\title{
Pharmacogenomic testing for
}

\section{angiotensin-converting enzyme inhibitors: getting ready for prime time}

"...after being a focal point of research for more than two decades, the clinical significance of the ACE insertion/deletion polymorphism remains to be established."

\section{KEYWORDS: angiotensin-converting enzyme inhibitor " atherosclerosis $\approx$ blood pressure $\approx$ hypertension $\approx$ pharmacogenomics}

Angiotensin-converting enzyme (ACE) inhibitors represent one of the great therapeutic success stories of cardiovascular pharmacotherapy. Clinical trials of ACE inhibitors have established that they are effective at reducing the risk of mortality and morbidity in diverse populations with cardiovascular diseases including hypertension, heart failure, myocardial infarction and stable coronary artery disease. This body of evidence has led to their widespread adoption in clinical practice. Interestingly, after more than three decades of investigation, our understanding of the pharmacological mechanisms by which ACE inhibitors exert their beneficial effects continues to evolve. Current data indicate that the biological effects of ACE inhibitors are far more intricate than the inhibition of the conversion of angiotensin I to angiotensin II, which results in a reduced activation of the angiotensin II type 1 receptor, the receptor that mediates the majority of angiotensin II's deleterious actions. Indeed, the physiological effects of ACE inhibitors include the modulation of several peptides as well as direct and indirect effects on specific receptors [1].

Despite these successes, a significant heterogeneity exists to the effectiveness and tolerability of ACE inhibitors [2,3]. Although data regarding the impact and cost of the interindividual variability of ACE inhibitors are limited from a population perspective, its consequences on individuals are clearly apparent in daily clinical practice. Indeed, because there are no available predictors of drug efficacy, their use is based on an empirical 'trial and error' approach. Furthermore, although some clinical characteristics can help identify individuals at higher risk of selected ACE inhibitor-induced adverse drug reactions [3], the information provided by these variables is generally not sufficient to identify individuals who do not require monitoring following the initiation of these drugs, or those in whom the risk of adverse drug reaction prohibits their use. Because a significant heritability to the renin-angiotensin-aldosterone system exists [4], many have proposed that genetic markers could be useful to identify individuals who are most likely to benefit from or tolerate these drugs.

In the August edition of Pharmacogenomics, Brugts et al. discussed fascinating data from the PERindopril GENEtic association study (PERGENE) [5], a substudy of the multicenter, randomized, placebo-controlled European trial on reduction of cardiac events with Perindopril in stable coronary artery disease (EUROPA) trial [6], which may lead to a more individualized use of perindopril in patients with stable coronary artery disease and, hopefully, of other ACE inhibitors in additional patient populations. In this large substudy of 8907 individuals, the investigators identified two SNPs (rs275651 and rs5182) from the AGTR1 gene, which encodes the angiotensin II type 1 receptor, as well as one SNP in the BDKRB1 gene (rs12050217), which encodes the bradykinin B1 receptor, that may help identify individuals who are most likely to experience a reduction in cardiovascular events when treated with perindopril. Moreover, the authors indicate that they observed similar trends in the Perindopril Protection against Recurrent Stroke Study (PROGRESS) trial, a study which evaluated the benefits of a perindopril-based treatment in patients with a previous stroke of transient ischemic attack [7].

The AGTR1 gene has been proposed by many as an important genetic determinant of the efficacy of renin-angiotensin-aldosterone

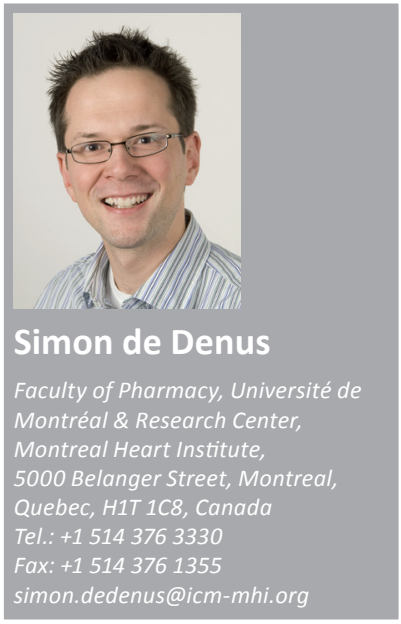


system modulators, in particular of ACE inhibitors and angiotensin II receptor blockers [8-10]. Nevertheless, results regarding the clinical impact of genetic polymorphisms of this gene, of which the A1166C polymorphism (rs5186) has probably been the most studied, have been inconsistent [11]. In this context, the investigation by Brugts, which was executed as part of a meticulously conducted multicenter randomized trial, makes a strong case for these two AGTR1 genetic polymorphisms as important players in ACE inhibitor pharmacogenomics. Validation from other cohorts is eagerly anticipated.

\section{"Some have rightfully argued that BP phenotyping in genomic studies is suboptimal..."}

The study by Brugts also provides potential novel insights into the pharmacology of ACE inhibitors. Indeed, their results regarding the $B D K R B 1$ gene are consistent with emerging data indicating that ACE inhibitors act as agonists of the bradykinin $\mathrm{B} 1$ receptor to release nitric oxide [1]. Although validation of this association in other cohorts of patients treated with ACE inhibitors and functional studies are required to better define the implications of these findings, they nonetheless illustrate how pharmacogenomics may not only lead to a more personalized pharmacotherapy, but also improve our understanding of diseases and their treatments. It is therefore hoped that leaders of other pharmacogenomic substudies from large clinical trials will launch investigations to validate this finding. Similarly, it would be of interest for the PERGENE investigators to validate other previously reported associations $[12,13]$. Consideration should also be given to the evaluation of the impact of the three SNPs identified in PERGENE and the risk of common adverse drug reactions induced by ACE inhibitors.

In a previous publication [14], the PERGENE investigators highlighted that their genotyping strategy incorporated key candidate genes from the renin-angiotensin-aldosterone system and bradykinin pathways, including the $A C E$ gene. It appears that none of the SNPs studied from that gene were associated with the effectiveness of perindopril, which is consistent with previous data from large clinical trials of ACE inhibitors that focused on the ACE insertion/deletion polymorphism [15,16]. It should be underlined that after being a focal point of research for more than two decades, the clinical significance of the ACE insertion/deletion polymorphism remains to be established. Nevertheless, we still cannot exclude the possibilty that other genetic polymorphisms in the $A C E$ gene may modulate ACE expression or activity and, ultimately, the response to ACE inhibitors. Of particular interest, the International Verapamil SR/Trandolapril study Genetic Substudy (INVEST GENES) group identified two SNPs (rs7213516 and rs4290) upstream of the ACE transcription start site that were strongly associated with ACE mRNA expression and the risk of cardiovascular events, particularly in individuals of African-American ancestry in whom these polymorphisms are more common [17]. The investigation of gene-gene or gene-environment interactions may also lead to the identification of clinically relevant SNPs within this gene [18].

Another aspect of the report by Brugts that deserves attention is that the three SNPs associated with the cardiovascular benefits of perindopril were not associated with its blood pressure (BP) reductions. Although many have argued that the benefits of ACE inhibitors in stable coronary artery disease are largely independent of their $\mathrm{BP}$ lowering effects, a topic of intense debate [19,20], it remains difficult to reconcile that genetic modulators of their cardiovascular benefits would not also be associated with the BP reductions of these highly effective antihypertensive drugs. Again, other previous multicenter pharmacogenomic studies of ACE inhibitors have had limited success identifying genetic predictors of BP reduction $[15,16]$. The challenge that the discovery of genetic modulators of BP reductions with antihypertensive drugs represents is well illustrated by the difficulties the genetic community has faced in identifying genetic determinants of $\mathrm{BP}$ and hypertension. We wonder whether this absence of genetic association with the BP reductions produced by antihypertensive drugs may, at least partly, be attributable to the difficulties of precisely characterizing the extremely variable phenotype that is BP, rather than a true absence of association.

Indeed, many factors contribute to the interindividual and intraindividual variability of BP, including circadian patterns of variation, medications and diet, which could modulate or mask genetic associations. Some have rightfully argued that $\mathrm{BP}$ phenotyping in genomic studies is suboptimal because it is generally limited to a few measurements at a given moment [18]. In light of the important variability of $\mathrm{BP}$ within an individual, such an approach is unlikely to fully characterize the BP phenotype for that 
individual or reductions in BP induced by antihypertensive drugs [21]. Blood pressure phenotyping can be improved by using $24 \mathrm{~h}$ ambulatory $\mathrm{BP}$ monitoring, which also minimizes measurement error and the white coat effect. Unfortunately, such fine phenotyping is difficult to perform in multicenter randomized trials that include thousands of individuals.

\section{"...international collaborations are now necessary so that hypertension pharmacogenomics can continue moving forward."}

Many other factors can confound the investigation of BP reductions in pharmacogenomic studies such as the time at which BP measurement is performed in regards to drug intake and drug compliance. The measurement of drug concentrations at the time of the BP measurement could be useful to take these factors into consideration. Limited data suggest that such an approach may also help identify genetic modulators of a drug's pharmacodynamics [9]. Another potential confounding factor is that drug treatments frequently vary between individuals in hypertension studies within a specific treatment group because the doses of the agents are adjusted or agents are used in different combinations to reach a target $\mathrm{BP}$ goal [11]. A final potential confounder of many hypertension pharmacogenomic studies is that patients already treated for this condition are commonly switched from their current medication to the study treatment without a wash-out period [22]. Hence, BP at baseline is not truly a 'baseline' measurement, which complicates the interpretation of BP changes during the study. Thus, a more meticulous phenotyping of $\mathrm{BP}$, while taking into consideration nongenetic factors contributing to BP variability, should be incorporated into future pharmacogenomic studies investigating BP lowering drugs such as ACE inhibitors.

In conclusion, the data reviewed by Brugts and colleagues represent an important step towards a more personalized use of ACE inhibitors. We believe that international collaborations are now necessary so that hypertension pharmacogenomics can continue moving forward. Ultimately, such international efforts will result in ACE inhibitor pharmacogenomics being finally ready for prime time.

\section{Financial \& competing interests disclosure}

The author has received research grants from AstraZeneca, Pfizer and Hoffmann LaRoche. The author is the Université de Montréal Beaulieu-Saucier Chair in Pharmacogenomics and is the recipient of a Personnel Award from the Heart and Stroke Foundation of Canada. He is also supported by the Fonds de la recherche en santé du Québec (FRSQ). The author has no other relevant affiliations or financial involvement with any organization or entity with a financial interest in or financial conflict with the subject matter or materials discussed in the manuscript apart from those disclosed.

No writing assistance was utilized in the production of this manuscript.

\section{Bibliography}

1 Erdos EG, Tan F, Skidgel RA:

Angiotensin I-converting enzyme inhibitors are allosteric enhancers of kinin B1 and B2 receptor function. Hypertension 55(2), 214-220 (2010)

2 Brunner M, Cooper-Dehoff RM, Gong Y et al: : Factors influencing blood pressure response to trandolapril add-on therapy in patients taking verapamil SR (from the International Verapamil SR/ Trandolapril [INVEST] Study). Am. J. Cardiol. 99(11), 1549-1554 (2007).

3 de Denus S, Tardif JC, White M et al:: Quantification of the risk and predictors of hyperkalemia in patients with left ventricular dysfunction: a retrospective analysis of the studies of left ventricular dysfunction (SOLVD) trials. Am. Heart J. 152(4), 705-712 (2006).
4 Vinck WJ, Fagard RH, Vlietinck R, Lijnen P: Heritability of plasma renin activity and plasma concentration of angiotensinogen and angiotensin-converting enzyme. J. Hum. Hypertens. 16(6), 417-422 (2002).

5 Brugts JJ, Boersma E, Simoons ML: Tailored-therapy of ACE-inhibitors in stable CAD: pharmacogenetic profiling of treatment benefit. Pharmacogenomics 11(8), 1115-1126 (2010).

6 Fox KM: Efficacy of perindopril in reduction of cardiovascular events among patients with stable coronary artery disease: randomised, double-blind, placebo-controlled, multicentre trial (the EUROPA study). Lancet 362(9386), 782-788 (2003).

7 PROGRESS collaborative group:

Randomised trial of a perindopril-based blood-pressure-lowering regimen among 6,105 individuals with previous stroke or transient ischaemic attack. Lancet 358(9287), 1033-1041 (2001).
8 de Denus S, Zakrzewski-Jakubiak M, Dube MP et al.: Effects of AGTRI A1166C gene polymorphism in patients with heart failure treated with candesartan. Ann. Pharmacother. 42(7), 925-932 (2008).

9 Kurland L, Hallberg P, Melhus H et al.: The relationship between the plasma concentration of irbesartan and the antihypertensive response is disclosed by an angiotensin II type 1 receptor polymorphism: results from the Swedish irbesartan left ventricular hypertrophy investigation vs. Atenolol (SILVHIA) trial. Am. J. Hypertens. 21(7), 836-839 (2008).

10 Su X, Lee L, Li X et al.: Association between angiotensinogen, angiotensin II receptor genes, and blood pressure response to an angiotensin-converting enzyme inhibitor. Circulation 115(6), 725-732 (2007). 
11 Nordestgaard BG, Kontula K, Benn M et al.: Effect of ACE insertion/deletion and 12 other polymorphisms on clinical outcomes and response to treatment in the LIFE study. Pharmacogenet. Genomics 20(2), 77-85 (2010).

12 Fan X, Wang Y, Sun K et al.: Polymorphisms of $A C E 2$ gene are associated with essential hypertension and antihypertensive effects of captopril in women. Clin. Pharmacol. Ther. 82(2), 187-196 (2007).

13 Lynch AI, Boerwinkle E, Davis BR et al.: Antihypertensive pharmacogenetic effect of fibrinogen $-\beta$ variant $-455 \mathrm{G}>\mathrm{A}$ on cardiovascular disease, end-stage renal disease, and mortality: the GenHAT study. Pharmacogenet. Genomics 19(6), 415-421 (2009).

14 Brugts JJ, De Maat MP, Boersma E et al.: The rationale and design of the perindopril genetic association study (PERGENE): a pharmacogenetic analysis of angiotensin- converting enzyme inhibitor therapy in patients with stable coronary artery disease. Cardiovasc. Drugs Ther. 23(2), 171-181 (2009).

15 Arnett DK, Davis BR, Ford CE et al: Pharmacogenetic association of the angiotensin-converting enzyme insertion/ deletion polymorphism on blood pressure and cardiovascular risk in relation to antihypertensive treatment: the genetics of hypertension-associated treatment (GenHAT) study. Circulation 111(25), 3374-3383 (2005).

16 Harrap SB, Tzourio C, Cambien F et al:: The $A C E$ gene I/D polymorphism is not associated with the blood pressure and cardiovascular benefits of ACE inhibition. Hypertension 42(3), 297-303 (2003).

17 Johnson AD, Gong Y, Wang D et al: Promoter polymorphisms in ACE (angiotensin I-converting enzyme) associated with clinical outcomes in hypertension. Clin. Pharmacol. Ther. 85(1), 36-44 (2009).
18 Franks PW: Identifying genes for primary hypertension: methodological limitations and gene-environment interactions. J. Hum. Hypertens. 23(4), 227-237 (2009).

19 Taylor R: Conundrum of the HOPE study: time of taking ramipril may account for lack of relation between blood pressure and outcome. BMJ 327(7416), 681-682, author reply 682 (2003).

20 Yusuf S, Bosch J, Sleight P: Preventing stroke with ramipril-authors' reply. BMJ 326(7379), 52 (2003).

21 Goyal D, Chong AY, Watson RL, Prasad N, Watson RD: Assessment of single versus twice daily dosing of ramipril by ambulatory blood pressure monitoring in patients similar to those included in the hope study. J. Hum. Hypertens. 21(7), 525-530 (2007).

22 Messerli FH, Weber MA: ALLHAt-all hit or all miss? Key questions still remain. Am. J. Cardiol. 92(3), 280-281 (2003). 